\title{
Control of electron-transfer in nanoimmunosensors by using polyclonal and monoclonal antibodies
}

\author{
Abdelmoneim Mars,,${ }^{[a, b]}$ Claudio Parolo,,${ }^{[a]}$ Alfredo de la Escosura-Muñiz, ${ }^{[a]}$ Noureddine Raouafi ${ }^{* b]}$ and Arben \\ Merkoçi ${ }^{*}[a, c]$ \\ a Catalan Institute of Nanoscience and Nanotechnology (ICN2), CSIC and The Barcelona Institute of Science and Technology, \\ Campus UAB, Bellaterra, 08193 Barcelona, Spain. \\ b Département de Chimie, Université de Tunis El-Manar, Faculté des Sciences, Campus universitaire de Tunis El-Manar, Tunis \\ 2092, Tunisia.
}

c ICREA, Barcelona, Catalonia, Spain.

* e-mail: arben.merkoci@icn.cat (Arben Merkoçi)

* e-mail: n.raouafi@ @st.rnu.tn (Noureddine Raouafi)

Received: ((will be filled in by the editorial sttaff)) Accepted: ((will be filled in by the editorial sttaff))
"This is the peer reviewed version of the following article:

A. Mars, C. Parolo, A. de la Escosura-Muñiz, N. Raouafi, A. Merkoçi, Control of electron-transfer in nanoimmunosensors by using polyclonal and monoclonal antibodies. Electroanalysis 2016, 28, 1795, which has been published in final form at https://doi.org/10.1002/elan.201500646

This article may be used for non-commercial purposes in accordance with Wiley Terms and Conditions for Use of Self-Archived Versions."

\begin{abstract}
The design and operation of biosensors is not trivial. For instance, variation in the output signal during monitoring of analytes cannot usually be controlled. Hence, if such control were possible, and could be triggered on demand, it would greatly facilitate system design and operation. Herein, we report the design of two types of voltamperometric immunosensors, in which the magnitude of the current output signal (differential pulse voltammetry [DPV]) can be increased or decreased as needed. The designed systems use monoclonal and polyclonal anti-human $\operatorname{IgG}$ antibodies, conjugated to monopodal ferrocene-modified gold nanoparticles that are casted onto screen-printed carbon electrodes ( $\mathrm{Ab} / \mathrm{mFcL} / \mathrm{AuNPs} / \mathrm{SPCEs}$ ). Upon addition of human IgG as antigen, the systems exhibit opposite responses according to the Ab: the current decreases when monoclonal $\mathrm{Ab}$ is used, whereas it increases when polyclonal $\mathrm{Ab}$ is used. We attributed the former response to inhibition of electron transfer (due to the formation of a protein layer), and the latter response, to a global increase in electron transfer (induced by the aggregation of gold nanoparticles). These effects were confirmed by studying a custom-made lipoic acid-based bipodal ligand, which confirmed that the increase in current is effectively induced by the aggregation of the modified nanoparticles (pAb/mFcL/AuNPs). Both sensors have large dynamic ranges, although the pAb-based one was found to be 3.3-times more sensitive. Tests of selectivity and specificity for ovalbumin, $\alpha$-lactalbumin and serum bovine albumin showed that the immunosensors are highly selective and specific, even in the presence of up to 1000 -fold levels of potentially competitive proteins. The limit of detection (LoD) for human IgG using the $\mathrm{pAb} / \mathrm{mFcL} / \mathrm{AuNP}$ bioconjugate was estimated to be $0.85 \mathrm{ng} / \mathrm{mL}$.
\end{abstract}

Keywords: Signal Control, Voltamperometric Biosensing, Immunosensor, Ferrocene, Gold Nanoparticles.

DOI: 10.1002/elan.((will be filled in by the editorial sttaff))

\section{Introduction}

Electrochemical biosensors are attracting major interest because of their potential for a myriad theranostics and environmental applications [1-3]. Moreover, they are good candidates for the basis of portable point-of-care devices, due to their inherent sensitivity, specificity, usability, miniaturisation and cost-effectiveness [4-6]. Gold nanoparticles (AuNPs) have been extensively used [7-10] in such sensors, owing to the fact that their surface Plasmon Resonance (SPR) electron field fully encompasses biomolecular interactions at nanoscale concentrations to ensure highly sensitive bioassays [11-13]. Size is another important factor for nanoparticles in electrochemical biosensors. For example, in a recent study, nanoparticles in the size range of 15 to $30 \mathrm{~nm}$ (similar to that of biomacromolecules) were recently shown to give the improved electrochemical results compared with other particles sizes [14-15]. 
Electroactive compounds, which are typically used as labels/transducers, can convert a biomolecular recognition event into measurable electrical signals. The amenability of ferrocene derivatives to this task has been demonstrated; in fact, they are quite useful for amperometric biosensors [16-17]. For instance, researchers have built an amperometric glucose sensor based on AuNPs modified with a thio- $\beta$ cyclodextrin/ferrocene inclusion complex and subsequently conjugated to glucose oxidase. This complex transducing system provides efficient electron shuttling, thereby enabling the oxidation of glucose at low potentials [18]. Very recently, ferrocene-functionalised peptide nanowires (size: $c a$. $100 \mathrm{~nm})$, coated with poly(diallyldimethylammonium chloride) and subsequently conjugated with gold nanoparticles and antibody, were used in a sensitive electrochemical immunosensor for an ELISA-like human IgG assay [19]. Upon addition of the analyte, the intensity of the current increase. Another amperometric immunosensor for the sensing of tumour necrosis factor $\alpha(\mathrm{TF} \alpha)$, in which the current intensity drops upon formation of the sandwich immunoassay, was prepared from a hydrogel made of ferrocenefunctionalised phenylalanine [20]. The authors attributed the decrease in current to the reduction of ferrocene by the ascorbic acid group attached to the second antibody of the immunosandwich. In another example, a system comprising ferrocene-branched chitosan deposited onto a glassy carbon electrode subsequently modified with AuNPs and hepatitis B surface (hBs) antibody has been used to detect hBs antigen ( $\mathrm{Ag})$ [21]. Gradual addition of hBs antigen induced a decrease in the DPV current, which was proportional to the amount of Ag added. The decrease in current was attributed to the hindrance of electron transfer from the ferrocene mediator to the electrode surface upon formation of the antibody-antigen immune complex. At higher concentrations of antigen, the immunocomplex acts as an inert kinetic barrier to electron transfer [22].

The aforementioned amperometric biosensors all demonstrate either of two responses: the detection leads to either an increase or a decrease in current. However, control over the response signal occurring in the device has not yet been achieved. Moreover, minor changes to the ferrocene compound can radically alter the sensor's electrochemical behaviour (i.e. potential-shifting vs. amperometric) of the biosensor, as recently reported by Mars et al. [23].

In this paper, we report a controllable design of two new electrochemical immunosensors for detection of human IgG as model analyte. Both sensors use gold nanoparticles modified with a monopodal ferrocene ligand ( $\mathrm{mFcL} / \mathrm{AuNPs}$ ) and biofunctionalized to either monoclonal antibodies (mAb/mFcL/AuNPs) or to polyclonal antibodies ( $\mathrm{pAb} / \mathrm{mFcL} / \mathrm{AuNPs}$ ). We showed that in both sensors the antigen binding leads to a change in the ferrocene oxidation current. However, and quite surprisingly, CV and DPV studies indicated that in the mAb-based sensor, the current decreases, whereas in the pAb-based sensor, it increases (Fig. 1). Further investigations proved that the latter response stems from aggregation of the NPs. The two biosensors have similar features, although the pAb-based one was more sensitive. The selectivity and specificity of the biosensors against other proteins were also evaluated.

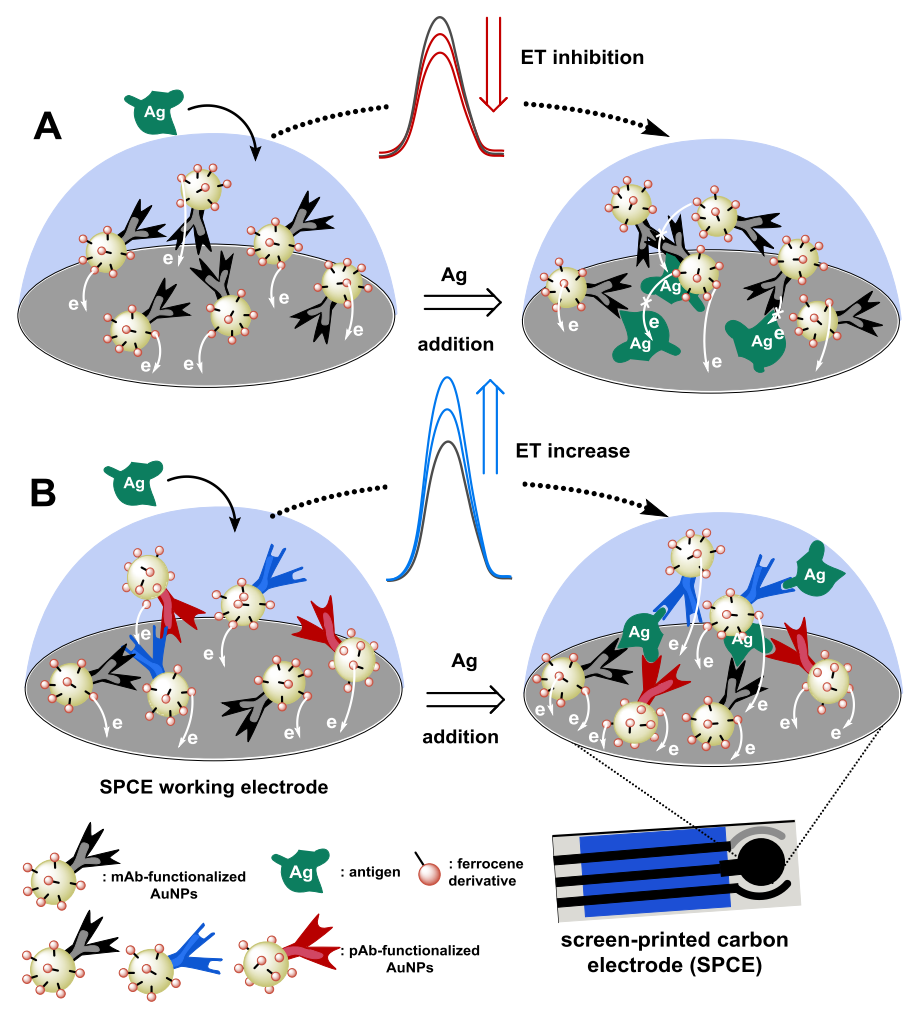

Fig. 1. Illustration of the responses observed for $\mathrm{mAb}$ - or $\mathrm{pAb}$ functionalised AuNPs using the same antigen human IgG and electrochemical immunosensor format human $\mathrm{IgG}$.

\section{Experimental}

\subsection{Materials and Apparatuses}

The following chemical and biochemical reagents were purchased from Sigma-Aldrich (Germany), and used as received without further purification: $\alpha$-lipoic acid $(\geq 98 \%) ; \quad 1$-ferrocenylmethanol $\quad(97 \%)$; dimethylaminopyridine (DMAP) (99\%); $\mathrm{HAuCl}_{4} \cdot 3 \mathrm{H}_{2} \mathrm{O} \quad(99.9 \%)$; dicyclohexylcarbimide (DCC) (99\%); sodium citrate trihydrate (99.0\%); terephthalaldehyde $(99 \%)$; sodium borohydride $(\geq$ $98 \%$ ); human $\operatorname{IgG}$ (hIgG, $\geq 95 \%$ from human serum); 
anti-human IgG monoclonal ( $\alpha$ hIgG produced in mouse) or polyclonal (produced in goat) antibodies; $\alpha$-lactalbumin ( $\geq 95 \%$ from human milk); ovalbumin ( $\geq 98 \%$ ); bovine serum albumin (98\%); silica gel (60 mesh) and $F_{254 n m}$ fluorescent silica-coated aluminium TLC plates. All the solvents used are analytical grade and were purchased from LabScan. The gold nanoparticles were synthesised by a modified Turkevich method (reduction of $\mathrm{HAuCl}_{4}$ with sodium citrate) [25].

All voltammetric experiments were performed in phosphate buffer solution ( $\mathrm{PBS}, \mathrm{pH}=7.4$ ) using $\mathrm{PC}$ controlled Metrohm Autolab PGSTAT101 or $\mu$ Autolab III electrochemical workstations. The experiments were designed and the data were collected using Nova software 1.10. Screen-printed carbon electrodes (SPCEs), printed on PET polymer foils using a DEK-248 screen printer (DEK International), were used for all the electrochemical

experiments. The SPCEs comprised a 3-mm diskshaped carbon working electrode, a printed $\mathrm{Ag} / \mathrm{AgCl}$ reference electrode and a carbon counter electrode and were printed on.

TEM micrographs were recorded using a Hitachi H600 transmission electron microscope. UV-visible spectroscopy was obtained on a SPECTRA ax M2e or a UNICO SpectroQuest 2800 spectrophotometer. The AuNP/antibody bioconjugates were purified on a ScanSpeed 1730R thermostatic centrifuge (LoboGene A/S, Danemark). All solutions were prepared in deionized water generated by a Milli-Q system (>18.2 $\mathrm{M} \Omega . \mathrm{cm}^{-1}$ ) from Millipore Inc. NMR spectra were recorded in a Bruker Advance 300 apparatus, in $\mathrm{CDCl}_{3}$ at $300 \mathrm{MHz}$ frequency. Chemical shifts are reported in ppm relative to tetramethylsilane (TMS) as internal reference.

\section{Results and Discussion}
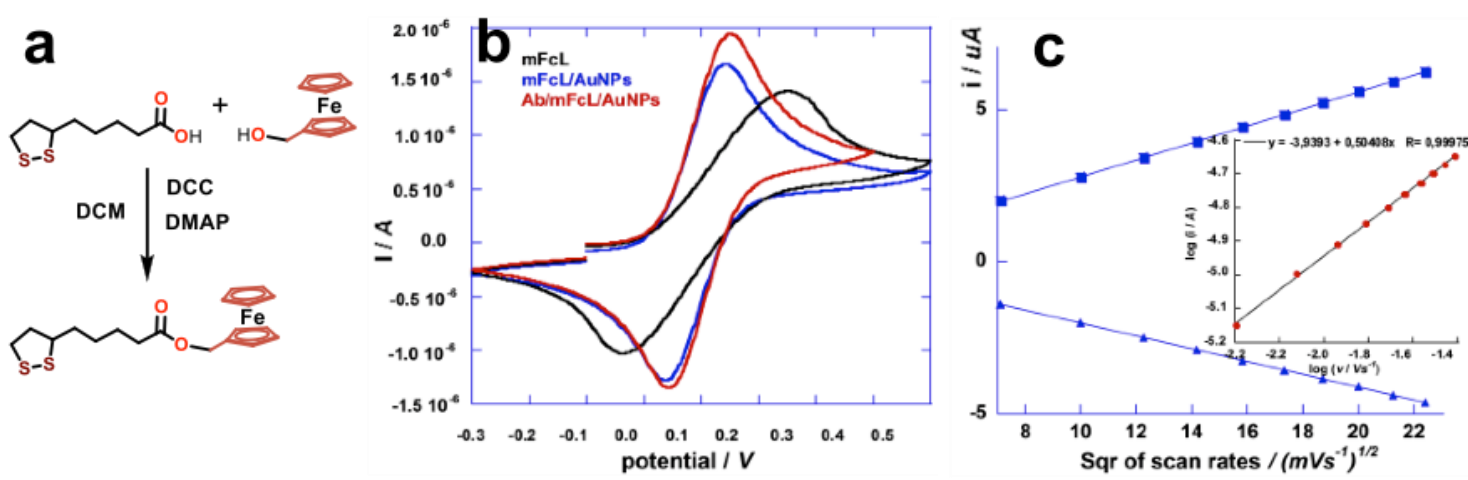

d
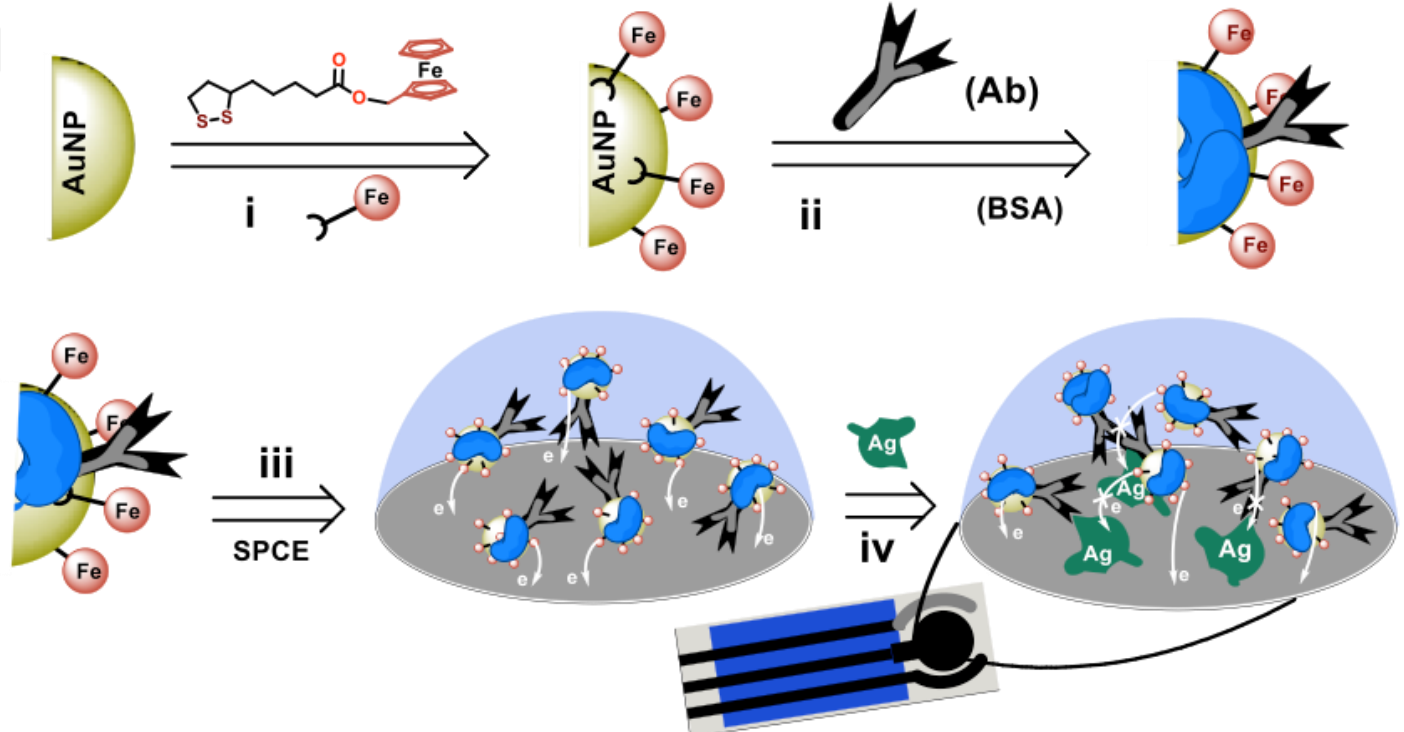

Fig. 2. (a) The DMAP-catalysed synthesis of mFcL, used to functionalise the AuNPs; (b) CV curves of (i: black) mFcL alone; (ii: blue) $\mathrm{mFcL} / \mathrm{AuNPs}$; and (iii) $\mathrm{Ab} / \mathrm{mFcL} / \mathrm{AuNPs}$; sweep rate: $50 \mathrm{mV} / \mathrm{s}$; working electrode: 3-mm carbon-printed disk, reference electrode: printed $\mathrm{AgCl} / \mathrm{Ag}$; (c) Plot of the oxidation and reduction peak currents vs. the square root of the scan rate (inset: plot of $\log i_{\mathrm{pa}}$ vs. logarithm of scan rate), and (d) Schematic of the fabrication of the Ab/mFcL/AuNP-based immunosensor: (i) functionalization of the AuNPs with $\mathrm{mFcL}$; (ii) conjugation with mAb or pAb, and surface blocking with BSA; (iii) casting onto SPCEs; and (iv) sensing of human IgG antigen. 


\subsection{Design of the immunosensors}

The AuNPs were first functionalised with $\mathrm{mFcL}$, which contains a 1,2-dithiolane moiety that has excellent affinity for gold [26]. The mFcL ligand was prepared via a Steglich DMAP-catalysed esterification of thioctic acid and ferrocenylmethanol, using DCC as condensing agent (Fig. 2a) [27]. The AuNPs were functionalized as described in Fig. 2a. The reaction with $\mathrm{mFcL}$ did not induce any colour change, whereas reaction with a similar but bipodal ligand induced a deep purple colouration, as previously reported [23]. Moreover UV-visible spectroscopy did not indicate any noticeable shift of the gold nanoparticle SPR band. The functionalization of AuNPs was confirmed by CV measurements of the $\mathrm{mFcL}$ before and after the addition of the AuNPs [23]. Upon addition, the shape and the current intensities of the $\mathrm{mFcL}$ changed slightly (Fig. 2b). A decrease in the peak-to-peak separation (i.e. from $\sim 280$ to $\sim 70 \mathrm{mV}$ ), and an increase in the oxidation peak and reduction peak currents, were observed, all suggesting improved system reversibility, and a better rate of electron transfer to the SPCE electrode, due to the AuNPs [18, 28-29]. The current increased by 2.2 times (Fig. S1 $\dagger$ in the Electronic Supplementary Information (ESI) file). Finally, to prepare the immunosensors for immediate use, the $\mathrm{mAb} / \mathrm{mFcL} / \mathrm{AuNP}$ and $\mathrm{pAb} / \mathrm{mFcL} / \mathrm{AuNP}$ bioconjugates were separately casted onto the working-electrode area of the SPCE. The different steps of the biosensor preparation are outlined in Fig. 2d.

The modification of $\mathrm{mFcL} / \mathrm{AuNPs}$ with the antibodies and $3 \%$ of BSA solution induced a slight anodic shift $(c a .20 \mathrm{mV})$ of the ferrocene $\mathrm{E}_{1 / 2}$ potential. The plot of the peak currents, recorded between 50 and $500 \mathrm{mV}$ of the square root of the scan rate, showed that the system is still reversible (Fig. 2c). Moreover, plotting the logarithm of peak current versus the logarithm of scan rate also showed straight lines with slopes of $0.50\left(R^{2}>0.999\right)$ for mAb, (inset in Fig. 2c). These facts suggest a predominantly diffusioncontrolled mass transfer of the modified gold particles from solution to the electrode inner diffusion layer [29-31].

\subsection{Immunosensing of human IgG}

\subsubsection{The mAb-based immunosensor}

We first studied the mAb-based immunosensor. Successive additions of human $\operatorname{IgG}(4.7 \mathrm{ng} / \mathrm{mL})$ induced a decrease in the DPV current (the ferrocene oxidation peak; see inset in Fig. 3b); when higher concentrations of human IgG $(47 \mathrm{ng} / \mathrm{mL})$ were used, the decreases in current were even larger. We attributed this effect to recognition of the human IgG by the mAb (see Figs. 3a and 3b, and the CVs in Fig. S2a). The DPV studies showed a continuous variation of the current up to a human IgG concentration of 600 $\mathrm{ng} / \mathrm{mL}$, proving that the immunosensor has a wide dynamic range. The calibration graph for the titration using CV is showed in Fig. S2b of the ESI file.

According to Qui et al. [22] and Ruther et al. [32], the decrease in current can be attributed to recognition of the antigen by the antibody-modified AuNPs, which results in formation of a dense protein layer. This layer hinders the completely inhibiting electron transfer from the ferrocene centre to the SPCE surface and consequently, causing a gradual decrease in the current that is proportional to the amount of $\mathrm{Ag}$ added.

The $\mathrm{mAb} / \mathrm{mFcL} / \mathrm{AuNP}$ was then analysed in the presence of high and low amounts of hIgG, by UV visible spectroscopy and transmission electron microscopy. As expected, due to the nature of the monoclonal antibody, no shifts in the UV-visible absorbance spectra were observed. The TEM images confirmed the mono-dispersity of the AuNPs solutions (Fig. S2).
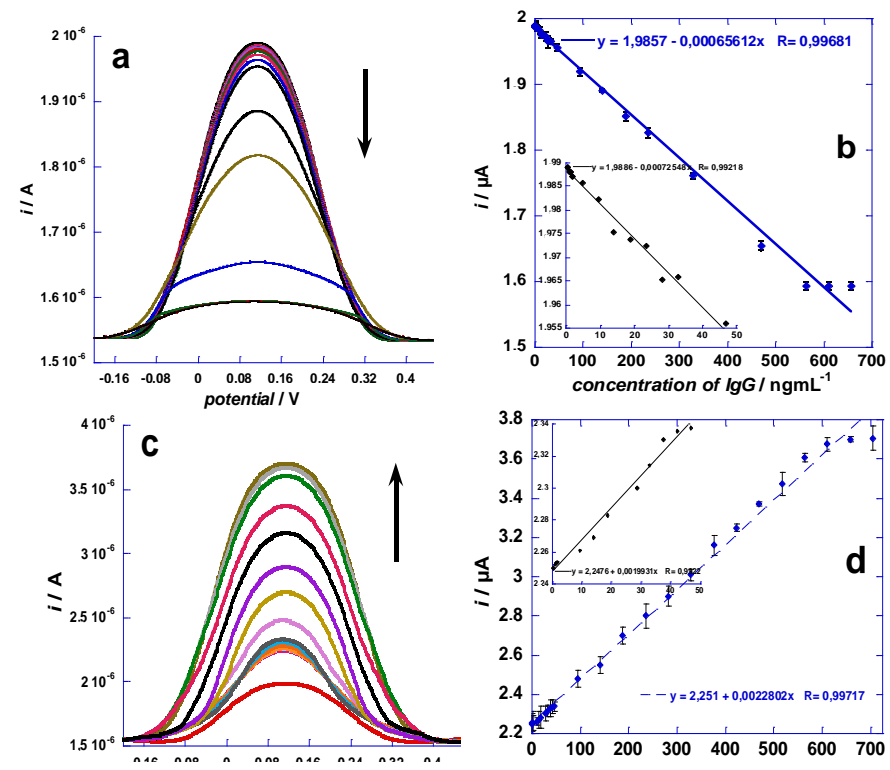

Fig. 3. Variations in DPV current in the mAb-based immunosensor, evidencing: (a) antigen/mAb recognition after progressive addition of the analytes; (b) decrease in current vs. IgG amount, and fitting curves (inset: effect of small antigen concentrations on the sensor response); (c) antigen/pAb recognition after progressive addition of the analytes; and (d) increase in current vs. human IgG amount, and fitting curves (inset: effect of small antigen concentrations on the sensor response).

\subsubsection{The pAb-based immunosensor}


Interestingly, gradual addition of human IgG to the pAb-based sensors led to proportional increases in DPV current (Fig. 3c): the current continued to rise linearly up to an human $\mathrm{IgG}$ concentration of $c a .700$ $\mathrm{ng} / \mathrm{mL}$ (Fig. 3d). In this case, the increase in current of the ferrocene oxidation peak can be reasonably attributed to the immune response (recognition of the antigen by the pAb). Similar results have been obtained by $\mathrm{CV}$ titration, CVs and the corresponding calibration plot are shown in Fig. S3.

We speculated that the aforementioned increase in current was due to the aggregation of individual $\mathrm{pAb} / \mathrm{mFcL} / \mathrm{AuNP}$ particulates when they are in the presence of the antigen. This is a reasonable explanation, given that the pAbs should recognize different epitopes of the antigen. We also speculated that the aggregation improved the deposition of the electroactive complexes onto the surface of the working electrode. Hence, the individual $\mathrm{pAb} / \mathrm{mFcL} / \mathrm{AuNPs}$ would contribute to the increase in the current generated from the ferrocene oxidation,

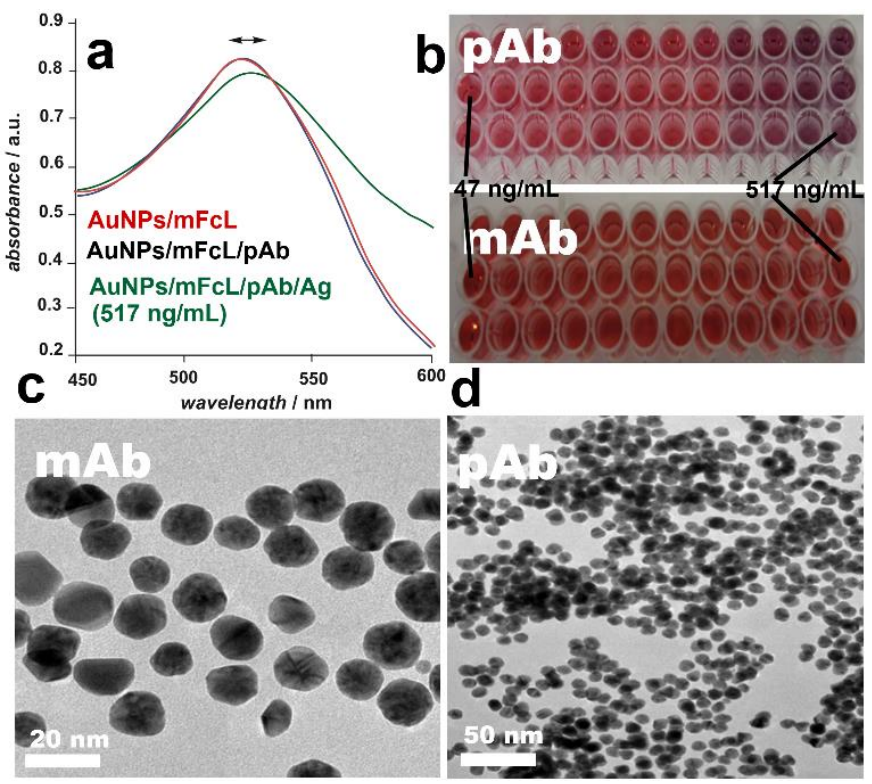

leading to improved electrochemical response [33]. In this scenario, the current will continue to rise until no free modified-AuNPs are available (i.e. all AuNPs are bound to antigen).

To verify our hypotheses, we first studied the aggregation of the $\mathrm{pAb} / \mathrm{mFcL} / \mathrm{AuNPs}$ by UV-visible spectroscopy. The UV-visible spectra showed that in

Fig. 4. (a) Shifts in the SPR band in the UV-visible spectra during recognition of the antigen by the pAb; (b) Visible colour change of the $\mathrm{pAb} / \mathrm{mFcL} / \mathrm{AuNP}$ conjugates upon gradual addition of human IgG (top), and unchanged colouration of the $\mathrm{mAb} / \mathrm{mFcL} / \mathrm{AuNP}$ suspension in the presence of antigen at various concentrations conjugate (bottom); (c) and (d) TEM micrograph revealing that the $\mathrm{pAb} / \mathrm{mFcL} / \mathrm{AuNPs}$ had aggregated at high concentrations of antigen (d), whereas the $\mathrm{mAb} / \mathrm{mFcL} / \mathrm{AuNPs}$ had remained monodisperse (c). the presence of low levels of antigen $(\sim 47 \mathrm{ng} / \mathrm{mL})$, the SPR band barely changed, whereas at high levels of antigen $(\sim 517 \mathrm{ng} / \mathrm{mL})$, it underwent a redshift of $c a$. $20 \mathrm{~nm}$ (Fig. 4a). Furthermore, unlike the mAb-based biosensor, in which the colouration of gold particles remained the same at all antigen concentrations (see Fig. 4b, inset), in the pAb-based biosensor, the colour of the particles changes from reddish to purple denoting the aggregation of the gold nanoparticles. Figure $4 \mathrm{~b}$ shows 96-well UV plates in which the antigen had been gradually and equally added to $\mathrm{mAb}$ and $\mathrm{pAb}$ solutions. For the $\mathrm{pAb}$ experiment, the resulting colour change was observable by the naked eye whereas no colour change was observed for the other Ab [34-37].

Using TEM, we then acquired further evidences to support our hypotheses. The TEM micrographs revealed that in the mAb-based sensor, in presence of high loading of the antigen, the particles had remained monodisperse (see Fig. 4d), whereas in the pAb-based sensor, the particles had indeed aggregated to form dense clusters of nanoparticles (see Fig. 4c). Results of TEM and UV-visible for $\mathrm{mAb}$-based biosensor are given Fig. S4.

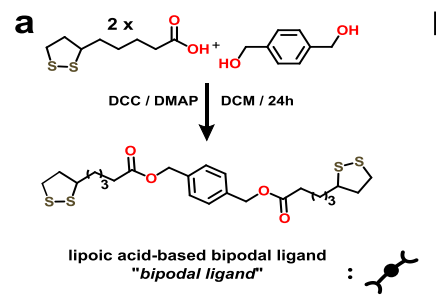

b
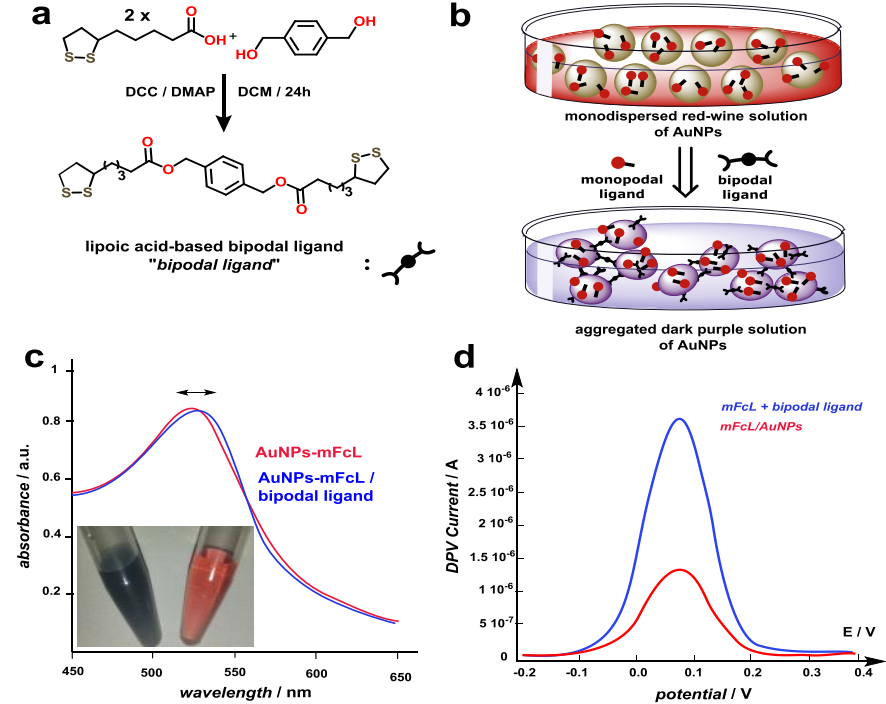

Fig. 5. a) Synthetic route to the lipoic acid-based bipodal ligand; b) Schematic of the aggregation of AuNPs containing the bipodal ligand; c) UV-Vis spectra for the reddish monodisperse mFcL/AuNPs (red plot), and the purple aggregates of $\mathrm{mFcL} / \mathrm{AuNPs}$ containing the bipodal ligand (blue plot) (inset: photos of each); and d) Plots of the DPV current for the reddish monodisperse mFcL/AuNPs (red plot), and the purple aggregates of $\mathrm{mFcL} / \mathrm{AuNPs}$ containing the bipodal ligand (blue plot)

\subsubsection{Provoking nanoparticle aggregation to induce} an increase in current 
In order to prove that the increase in current that we observed in the pAb-based immunosensor was due to aggregation of the $\mathrm{pAb} / \mathrm{mFcL} / \mathrm{AuNPs}$ triggered by recognition of the antigen by the pAbs, we studied an artificial organic linker that causes AuNPs to aggregate (i.e. that would cause an increase in DPV current). We designed and prepared a bipodal lipoic acid-based ligand bearing a lipoic unit on each side. We chose lipoic acid to avoid ligand exchange at the gold surface. The synthesis of the bipodal ligand is outlined in Figure 5a. As depicted in Figure 5b, the ligand caused the AuNPs to aggregate, which led to interaction between their individual SPR bands and consequently, to plasmonic coupling. As a result, unlike the reddish $\mathrm{mFcL} / \mathrm{AuNPs}$, the $\mathrm{mFcL} / \mathrm{AuNPs}$ containing the bipodal ligand were purple, exhibiting a $10-\mathrm{nm}$ redshift in their UV-Vis spectrum relative to that of the former (Figure 5c) [34-37]. Moreover, when $1 \mu \mathrm{L}$ of a conc. ethanolic solution of the bipodal ligand was added to $50 \mu \mathrm{L}$ of $\mathrm{mFcL} / A u N P s$, the particles aggregated, provoking an approximately 3-fold increase in the DPV current under the same conditions previously used (Figure $5 \mathrm{~d}$ ). We can speculated that electron transfer from a ferrocene moiety, sandwiched between aggregated nanoparticles, to the electrode surface is improved by proximity of the former to multiple gold nanoparticles. Furthermore, said improvement could be attributed to the plasmonic coupling, although we did not observe any effect on the ferrocene oxidation potential.

\subsubsection{Monoclonal vs. polyclonal}

We run each experiment with anti-human IgG in triplicate. The correlation curve for both antibodies are straight lines (coefficient of correlation $\mathrm{r}^{2}>0.996$ ), which confirmed good reproducibility of the measurements [38].

Based on the calibration plots, the mAb-based immunosensor exhibited a dynamic range of 4.7 to $\sim 600 \mathrm{ng} / \mathrm{mL}$, a sensitivity of $0.6610^{-3} \mu \mathrm{A} \cdot \mathrm{mL} / \mathrm{ng}$ and a limit of detection (LoD) of $1.75 \mathrm{ng} / \mathrm{mL}$. In contrast, the pAb-based immunosensor showed slightly better performance, with a dynamic range of 4.7 to $\sim 650$ $\mathrm{ng} / \mathrm{mL}$, a sensitivity of $2.2810^{-3} \mu \mathrm{A} \cdot \mathrm{mL} / \mathrm{ng}$; and an LoD of $0.87 \mathrm{ng} / \mathrm{mL}$. Furthermore, it showed a LoQ of $2.9 \mathrm{ng} / \mathrm{mL}$.

Comparing the slopes of the two biosensors, the pAb-based immunosensor appears to be $\sim 3.5$-fold more sensitive. Moreover, it shows a chromogenic response and cheaper antibodies. This result sharply contrasts with earlier findings from our group (we previously reported that a pAb-based, potentialshifting biosensor was less sensitive than its monoclonal analogue [23]) and others [31].

\subsection{Selectivity and specificity of the immunoassay}

We then sought to assess the selectivity and specificity of the pAb-based immunosensor, as these are two important features for any biosensor. Several tests were run to prove that the observed DPV amperometric response was indeed due to formation of the immune complex and to rule out the possibility that the variations in current were due to non-specific protein adsorption on the surface of the AuNPs. BSA, $\alpha$-LA and OVA were separately tested as potentially interfering proteins to the antigen adsorption. The selectivity tests were conducted by measuring the amperometric response of the pAb-based immunosensor against a 1000-fold concentration of each test protein compared to that given by $c a .100 \mathrm{pg}$ $(1 \mu \mathrm{L}, 94 \mathrm{ng} / \mathrm{mL})$ of the antigen. The addition of 100 ng $(1 \mu \mathrm{L}, 100 \mu \mathrm{g} / \mathrm{mL})$ of $\alpha$-LA showed no effect (Fig. $6 a)$ on the biosensor response, whereas the addition of $94 \mathrm{pg}$ of human IgG induced an approximately $25 \%$ increase in DPV current; similar results were obtained with BSA and OVA (Fig. S5) [42]. Based on the results (summarised in Fig. 6b.), we concluded that the
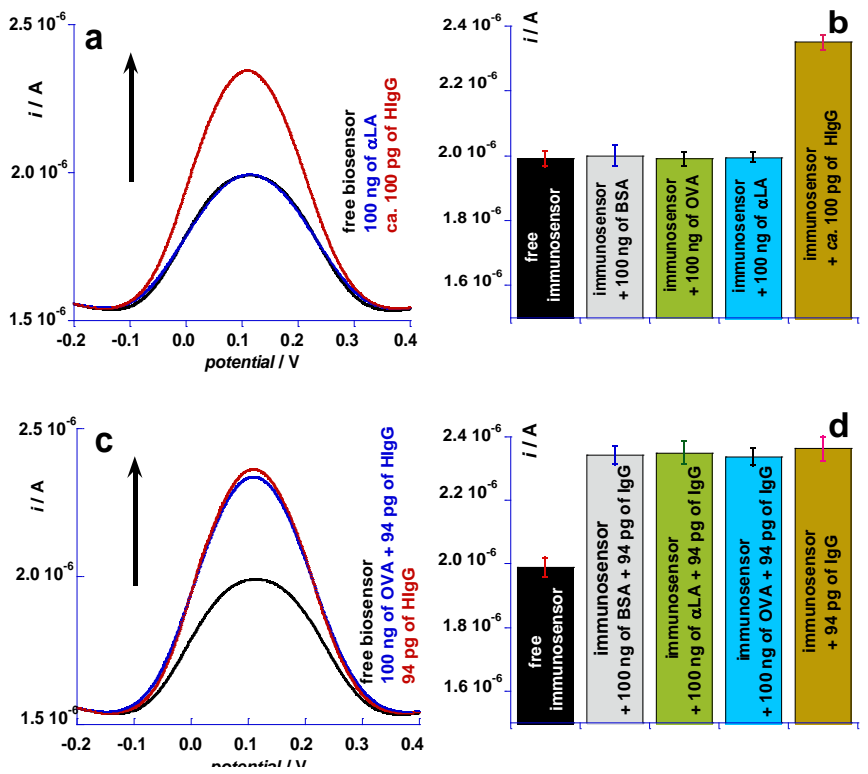

Fig. 6. Selectivity and specificity of the pAb-based immunosensor for human $\mathrm{IgG}$, as tested against possible interfering proteins: (a) DPV current response of the sensor to $\alpha$ LA protein (see ESI for the analogous tests with BSA and OVA); (b) Histograms summarising the sensors' relative selectivity for human IgG, BSA, $\alpha$-LA and OVA; (c) DPV current response to antigen and to a mixture of antigen and a 1000-fold excess of OVA protein; and (d) amperometric response of the immunosensor to human $\operatorname{IgG}$ and BSA, human IgG and $\alpha-\mathrm{LA}$, and human IgG and OVA.

pAb-immunosensor is highly selective for human IgG protein. 
We performed additional experiments to demonstrate the specificity of the pAb-based immunosensor for human IgG. The results are summarised in the histogram of Fig. 6d. To evaluate its specificity for the antigen over OVA, its responses to the antigen alone and to the antigen in presence of OVA were measured and then compared. The electrochemical signals were very similar in presence or absence of OVA (difference: 1.1\%), demonstrating the very high specificity to human IgG (Fig. 6b). Each measurement was run in triplicate to ensure reproducibility (the analogous results from BSA and $\alpha$-LA are given in Fig. S6) [37].

\section{Conclusions}

We have designed and fabricated two immunosensors based on ferrocene-modified gold nanoparticles functionalised with either monoclonal $(\mathrm{mAb})$ or polyclonal (pAb) anti-human IgG antibodies. We have demonstrated that the amperometric response of these immunosensors depends on the antibodies used. In both sensors, the antigen binding leads to a change in the ferrocene oxidation current. However, and quite surprisingly, in the mAb-based sensor, immunosensing leads to a decrease in current, whereas in the pAb-based sensor, it leads to an increase in current (Figure 1). The former can be explained by the formation of a protein layer that inhibits electron transfer from the ferrocene moieties to the electrode surface, whereas the latter can be explained by agglomeration of the AuNPs, which enhanced the electron transfer. Further investigations proved that the latter response stems from aggregation of the NPs. The pAb-based sensor performed better, exhibiting greater sensitivity (2.28 $\left.10^{-3} \mu \mathrm{A} \cdot \mathrm{mL} / \mathrm{ng}\right)$ and a higher $\operatorname{LoD}(0.87 \mathrm{ng} / \mathrm{mL})$. Given their wide detection range, these label-free, fast and versatile sensors can be adapted to many different analytes, making them useful for diagnostic, environmental and safety applications.

\section{Acknowledgments}

ICN2 acknowledges support from the Severo Ochoa Program (MINECO, Grant SEV- 2013-0295). Authors wish to thank Generalitat de Cataluña for SGR support and the MHESR, University of Tunis ElManar and the LCAE laboratory for research and travel funds granted to AMM. MEC (Spain) for MAT2011-25870 grant is also acknowledged.
[1] C. R. Ispas, G. Crivat and S. Andreescu, Anal. Lett., 2012, 45, 168-186.

[2] J. Zhang, S. Song, L. Wang, D. Pan and C. Fan, Nat. Protoc., 2007, 2, 2888-2895.

[3] N. S. Lynn, H. Šípová, P. Adam and J. Homola, Lab Chip, 2013, 13, 1413-1421

[4] W. Schuhmann and K. Habermüller «Miniaturisation of Biosensors» in «Electrochemical Microsystem Technologies» Ed. J.W. Schultze, T. Osaka, M. Datta, CRC Press, London, New York, 2002, Chap. 16, 409428.

[5] J. Wang, Biosens. Bioelectron., 2006, 21, 1887-1892.

[6] A. P. F. Turner, Chem. Soc. Rev., 2013, 42, 3184-3196.

[7] M. Maltez-Da Costa, A. de la Escosura-Muniz and A. Merkoçi, Electrochem. Commun., 2010, 12, 1501-1504.

[8] T. Špringer and J. Homola, Anal. Bioanal. Chem., 2012, 404, 2869-2875.

[9] S. Zeng, K.-T. Yong, I. Roy, X.-Q. Dinh, X. Yu and F. Luan, Plasmonics, 2011, 6, 491-506 and reference therein.

[10] J. M. Pingarron, P. Yanez-Sedeno and A. GonzalezCortés, Electrochim. Acta, 2008, 53, 5848-5866.

[11] S. Eustis and M.A. El-Sayed, Chem. Soc. Rev., 2006, 35, 209-217.

[12] K. V. Singh, A. M. Whited, Y. Ragineni, T. W. Barrett, J. King and R. Solanki, Anal. Bioanal. Chem., 2010, 397, 1493-1502.

[13] V. Bhalla, S. Carrara, P. Sharma, Y. Nangia, C. R. Suri, Sensor. Actuat. B, 2012, 161, 761-768.

[14] N. Wangoo, J. Kaushal, K.K. Bhasin, K.K. Mehta and C.R. Suri, Chem. Commun., 2010, 46, 5755-5757.

[15] A. de la Escosura-Muñiz, C. Parolo, F. Maran and A. Mekoçi, Nanoscale, 2011, 3, 3350-3356.

[16] M. Liu, L. Wang, J. Deng, Q. Chen, Y. Li, Y. Zhang, H. Li and S. Yao, Analyst, 2012, 137, 4577-4583.

[17] A. Chaubey and B.D. Malhotra, Biosens. Bioelectron., 2002, 17, 441-456.

[18] M. Chen and G. Diao, Talanta, 2009, 80, 815-820.

[19] Y. Ding, D. Li, B. Li, K. Zhao, W. Du, J. Zheng and M. Yang, Biosens. Bioelectron., 2013, 48, 281-286.

[20] Y. Hou, T. Li, H. Huang, H. Quan, X. Miao and M. Yang, Sensor. Actuat. B, 2013, 182, 605-609.

[21] J.-D. Qiu, R.-P. Liang, R. Wang, L.-X. Fan, Y.-W. Chen and X.-H. Xia, Biosens. Bioelectron., 2009, 25, $852-857$.

[22] J.-D. Qui, H. Huang and R.-P. Liang, Microchim. Acta, 2011, 174, 97-105.

[23] A. Mars, C. Parolo, N. Raouafi, K. Boujlel and A. Merkoçi, J. Mater. Chem. B, 2013, 1, 2951-2955.

[24] C. S. Lim, A. Ambrosi, Z. Sofer and M. Pumera, Nanoscale, 2014, 6, 7391-7396.

[25] J. Kimling, M. Maier, B. Okenve, V. Kotaidis, H. Ballot and A. Plech, J. Phys. Chem. B, 2006, 110, 1570015707.

\section{References}


[26] R. Sahli, C. Fave, N. Raouafi, K. Boujlel, B. Limoges and B. Schollhörn, Langmuir, 2013, 29, 53605368.

[27] B. Neises and W. Steglich, Angew. Chem. Int. Ed., 1978, 17, 217-222.

[28] V. V. Shumyantseva, S. Carrara, V. Bavastrello, D. J. Riley, T. V. Bulko, K. G. Skryabin, A. I. Archakov and C. Nicolni, Biosens. Bioelectron., 2005, 21, 852-857.

[29] A.S. Barnes, E.I. Rogers, I. Streeter, L. Aldous, C. Hardcore and R.G. Compton, J. Phys. Chem. B, 2008, 112, 7560-7565.

[30] E. Saint-Aman, M. Ungureanu, T. Visan nd J. C. Mouter, Electrochim. Acta, 1997, 42, 1829-1837.

[31] R. Sahli, N. Raouafi, E. Maisonhaute, K. Boujlel and B. Schollhörn, Electrochim. Acta, 2012, 63, 228-231.

[32] R. E. Ruther, Q. Cui and R. J. Hamers, J. Am. Chem. Soc., 2013, 135, 5751-5761.

[33] H. Ju, X. Zhang and J. Wang, NanoBiosensing: Principles, Development and Application, Springer, 2011, Chap. 2, 39-84.

[34] C. Leng, G. Lai, F. Yan and H. Ju, Anal. Chim. Acta, 2010, 666, 97-101.

[35] A. Kumar and P. Paul, Anal. Methods, 2014, 6, 45514558.

[36] P.K. Jain and M.A. El-Sayed, Chem. Phys. Letters, 2010, 487, 153-164.

[37] H. Chen, J. Zhang, X. Liu, Y. Gao, Z. Ye, and G. Li, Anal. Methods, 2014, 6, 2580-2585.

[38] J. C. Vidal, L. Bonel, P. Duato and J. R. Castillo, Anal. Methods, 2011, 3, 977-984.

[39] R. Chai, R. Yuan, Y. Chai, C. Ou, S. Cao and X. Li, Talanta, 2008, 74, 1330-1336.

[40] Y. Zhuo, R. Yuan, Y. Chai, D. Tang, Y. Zhang, N. Wang, X. Li and Q. Zhu, Electrochem. Commun., 2005, 7, 355-360.

[41] X. Xue, D. Wei, R. Feng, H. Wang, Q. Wei and B. Du, Anal. Methods, 2013, 5, 4159-4164.

[42] E. Alipour, H. Ghourchian and S. M. Boutorabi, Anal. Methods, 2013, 5, 4448-4453. 